\title{
Enhanced Photon Extraction from a Nanowire Quantum Dot Using a Bottom-Up Photonic Shell
}

Jeannin, Mathieu; Cremel, Thibault; Häyrynen, Teppo; Gregersen, Niels; Bellet-Amalric, Edith; Nogues, Gilles; Kheng, Kuntheak

Published in:

Physical Review Applied

Link to article, DOI:

10.1103/PhysRevApplied.8.054022

Publication date:

2017

Document Version

Publisher's PDF, also known as Version of record

Link back to DTU Orbit

Citation (APA):

Jeannin, M., Cremel, T., Häyrynen, T., Gregersen, N., Bellet-Amalric, E., Nogues, G., \& Kheng, K. (2017).

Enhanced Photon Extraction from a Nanowire Quantum Dot Using a Bottom-Up Photonic Shell. Physical Review Applied, 8(5), [054022]. https://doi.org/10.1103/PhysRevApplied.8.054022

\section{General rights}

Copyright and moral rights for the publications made accessible in the public portal are retained by the authors and/or other copyright owners and it is a condition of accessing publications that users recognise and abide by the legal requirements associated with these rights.

- Users may download and print one copy of any publication from the public portal for the purpose of private study or research.

- You may not further distribute the material or use it for any profit-making activity or commercial gain

- You may freely distribute the URL identifying the publication in the public portal 


\title{
Enhanced Photon Extraction from a Nanowire Quantum Dot Using a Bottom-Up Photonic Shell
}

\author{
Mathieu Jeannin, ${ }^{1}$ Thibault Cremel, ${ }^{2}$ Teppo Häyrynen, ${ }^{3}$ Niels Gregersen, ${ }^{3}$ Edith Bellet-Amalric, ${ }^{2}$ \\ Gilles Nogues, ${ }^{1, *}$ and Kuntheak Kheng ${ }^{2}$ \\ ${ }^{1}$ Université Grenoble Alpes, CNRS, Institut Néel, "Nanophysique et semiconducteurs" group, \\ F-38000 Grenoble, France \\ ${ }^{2}$ Université Grenoble Alpes, CEA, INAC, PHELIQS, "Nanophysique et semiconducteurs" group, \\ F-38000 Grenoble, France \\ ${ }^{3}$ DTU Fotonik, Department of Photonics Engineering, Technical University of Denmark, \\ Ørsteds Plads, Building 343, DK-2800 Kongens Lyngby, Denmark
}

(Received 2 March 2017; published 10 November 2017)

\begin{abstract}
Semiconductor nanowires offer the possibility to grow high-quality quantum-dot heterostructures, and, in particular, CdSe quantum dots inserted in $\mathrm{ZnSe}$ nanowires have demonstrated the ability to emit single photons up to room temperature. In this paper, we demonstrate a bottom-up approach to fabricate a photonic fiberlike structure around such nanowire quantum dots by depositing an oxide shell using atomiclayer deposition. Simulations suggest that the intensity collected in our NA $=0.6$ microscope objective can be increased by a factor 7 with respect to the bare nanowire case. Combining microphotoluminescence, decay time measurements, and numerical simulations, we obtain a fourfold increase in the collected photoluminescence from the quantum dot. We show that this improvement is due to an increase of the quantum-dot emission rate and a redirection of the emitted light. Our ex situ fabrication technique allows a precise and reproducible fabrication on a large scale. Its improved extraction efficiency is compared to state-of-the-art top-down devices.
\end{abstract}

DOI: 10.1103/PhysRevApplied.8.054022

\section{INTRODUCTION}

Controlling and enhancing the spontaneous emission of quantum emitters is one of the current key issues in the field of nanophotonics. Semiconductor quantum dots (QDs) are considered to be promising and efficient single-photon emitters for quantum-optics applications [1-6]. Over the past few years, several approaches have been pursued to control their emission properties, from the use of photonic crystals $[7,8]$ to top-down photonic wires [9-11] and trumpets $[12,13]$. These strategies are based on the early work of Purcell [14] which demonstrated that the spontaneous emission of an emitter can be modified by engineering its electromagnetic environment. They rely on a waveguiding approach to increase the coupling between a well-defined propagating optical mode and the QD, while simultaneously reducing the coupling between the QD and background radiation modes, offering control of both the optical mode profile and the QD spontaneous emission rate.

In this context, the interest of the dot-in-a-nanowire configuration fabricated using bottom-up methods naturally arises because it provides a simple way to ensure the centering of a single quantum emitter in the photonic structure [15-17]. The bottom-up fabrication method also avoids heavy processing, like etching the semiconducting material, that is often detrimental to the optical properties of

\footnotetext{
"gilles.nogues@neel.cnrs.fr
}

the QDs. However, the main realizations up to now concern III-V semiconductors [15-17], limiting the operation range to the cryogenic temperature. Tackling this issue, the potential of II-VI materials, in particular, CdSe QDs inserted inside $\mathrm{ZnSe}$ nanowires (NWs), has been demonstrated in previous studies. They allow for robust hightemperature single-photon emission using heteroepitaxial [18] or homoepitaxial [19] nanowire growth. Contrary to all of the aforementioned systems where the photonic wire structure has a diameter comparable to the wavelength $\lambda / n$ of the guided light which allows for highly efficient coupling to the $\mathrm{HE}_{11}$ mode [20], the diameter of the II-VI NW embedding the QD $(\sim 20 \mathrm{~nm})$ is much smaller than the wavelength of the emitted light $(530 \mathrm{~nm})$. It leads to light emission predominantly into nonguided radiation modes and a low collection efficiency. An additional fabrication effort has thus to be made to ensure an efficient coupling to the collection optics.

In a previous report [21] we theoretically investigated the potential of using an oxide-shell deposition on a bare $\mathrm{ZnSe}$ NW to form a thick photonic wire structure. In this article, we experimentally demonstrate the use of atomic-layer deposition (ALD) to fabricate a conformal aluminum-oxide $\left(\mathrm{Al}_{2} \mathrm{O}_{3}\right)$ shell around $\mathrm{ZnSe} \mathrm{NWs}$ containing a single CdSe QD. We show that the oxide shell drastically enhances the light intensity emitted by the QD, and we use time-resolved microphotoluminescence to systematically study the effect of the shell thickness on the nanowire quantum dot 
(NWQD) emission rate. Our results are compared to numerical simulations accounting for the real NW geometry, evidencing the different physical mechanisms leading to the enhancement of the spontaneous emission from the QD and to the improved light collection from the emitting structure.

\section{PRINCIPLES OF OPERATION}

To illustrate the effect of the NW and its surrounding medium on the QD emission rate, let us consider a QD placed inside an infinitely long cylinder as illustrated in Fig. 1(a) radiating a field at a wavelength $\lambda$. The cylinder is made of a dielectric material (refractive index $n$ ) and has a diameter $d$. We first consider a dipole orientation perpendicular to the NW axis in order to use the NW as a propagation medium for the emitted light. In the limit where $d \ll \lambda / n$, the dielectric screening effect [11] reduces the spontaneous emission rate $\gamma$ by a factor

$$
\frac{\gamma}{\gamma_{0}}=\frac{4}{n\left(n^{2}+1\right)^{2}}
$$

where $\gamma_{0}$ is the radiative emission rate in the bulk material of index $n$ [22]. For a ZnSe cylinder $\left(n_{\mathrm{ZnSe}}=2.68\right.$ at $\lambda=530 \mathrm{~nm}$ ), the screening factor is $\sim 1 / 45$. If the NW is surrounded by a shell of refractive index $n_{s}$ instead of vacuum, Eq. (1) remains valid by replacing $n$ with the index contrast $n / n_{s}$. For an $\mathrm{Al}_{2} \mathrm{O}_{3}$ surrounding medium $\left(n_{s}=1.77\right)$, the screening factor becomes $\sim 1 / 4.1$, resulting in an order-of-magnitude larger radiative rate.

In addition to changing the dielectric screening, the $\mathrm{Al}_{2} \mathrm{O}_{3}$ shell also influences the guiding of light along the NW. We have computed the total emission rate $\gamma$ and the emission rate $\gamma_{\mathrm{HE} 11}$ into the fundamental $\mathrm{HE}_{11}$ waveguide mode from a radial dipole as a function of the shell thickness $t_{s}$ [see Fig. 1(a)] using a semianalytical approach [23] combined with an efficient nonuniform discretization scheme in $k$ space [24]. The results are plotted in Fig. 1(b). We observe that the shell thickness of $\sim 120 \mathrm{~nm}$ not only leads to an increased total emission rate, it also allows for
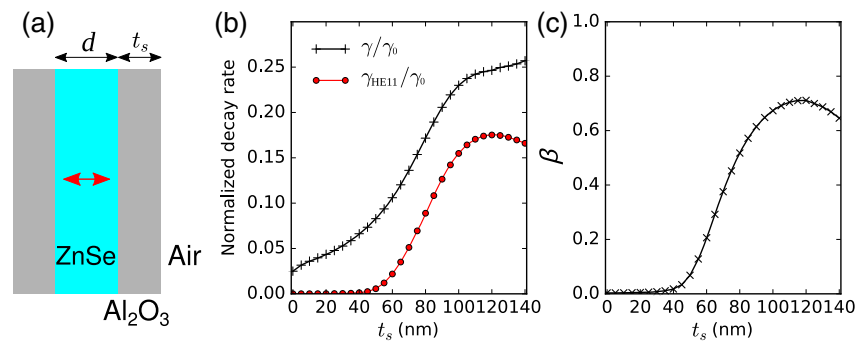

FIG. 1. (a) Geometry of the infinite NW. (b) Total spontaneous emission rate (black plus symbol) and spontaneous emission rate into the first guided mode $\mathrm{HE}_{11}$ (red circle) as a function of shell radius for a radial dipole. (c) Fraction $\beta$ of power radiated into the $\mathrm{HE}_{11}$ mode. confinement of the fundamental $\mathrm{HE}_{11}$ mode to the coreshell NW leading to a preferential coupling of the emitted light to this mode. Figure 1(c) presents the spontaneous emission $\beta$ factor representing the fraction $\beta=\gamma_{\mathrm{HE} 11} / \gamma$ of emitted light coupled to the $\mathrm{HE}_{11}$ mode. We observe indeed that up to $71 \%$ of the emitted light is coupled to this mode for $t_{s}=120 \mathrm{~nm}$. The dipole thus becomes coupled to the equivalent of a monomode photonic wire [9-11,15-17] paving the way to the control of its far-field radiation pattern.

\section{SAMPLE FABRICATION}

Our emitters are CdSe QDs embedded inside a ZnSe NW with a thin, epitaxial passivation $\mathrm{Zn}_{0.83} \mathrm{Mg}_{0.17} \mathrm{Se}$ shell grown around the NW. They are grown by molecular beam epitaxy on a GaAs(111)B substrate. A ZnSe buffer layer is first grown on the GaAs substrate, after which a thin layer of $\mathrm{Au}$ (less than one monolayer thick) is evaporated on the sample surface and dewetted at $510^{\circ} \mathrm{C}$ to form small ( 10-nm diameter) Au droplets that serve as a catalyst for the NW growth. The substrate temperature is then set at $400{ }^{\circ} \mathrm{C}$ and a flux of $\mathrm{Zn}$ and Se atoms with an excess of Se is used, inducing preferential growth of vertical $\mathrm{ZnSe}$ NWs. The NWs are in the wurtzite phase and their diameter is the same as the droplet ( $\sim 10-\mathrm{nm}$ diameter). The thickness of the initial Au layer is chosen to ensure a low NW density ( $\leq 1 \mathrm{NW}$ per $\mu \mathrm{m}^{2}$ ). After the growth of a $400-\mathrm{nm}$-high NW, the atom fluxes are stopped to allow the evacuation of residual Se atoms inside the droplet. Then, the QD is grown under a flux of $\mathrm{Cd}$ and $\mathrm{Se}$ atoms for $20 \mathrm{~s}$. The fluxes are interrupted again before the $\mathrm{ZnSe}$ growth is resumed, resulting in an expected QD height of 2-3 nm inserted in a 700-nm-high NW. Finally, an epitaxial $\mathrm{Zn}_{0.83} \mathrm{Mg}_{0.17} \mathrm{Se}$ shell (5-nm thick) is grown around the NW at $220^{\circ} \mathrm{C}$. A scanning electron microscope (SEM) image of such a $\mathrm{CdSe} / \mathrm{ZnSe} / \mathrm{Zn}_{0.83} \mathrm{Mg}_{0.17} \mathrm{Se}$ core-shell NWQD system is presented in Fig. 2(a). The flag-shape termination of the NW is formed during the growth of the $\mathrm{Zn}_{0.83} \mathrm{Mg}_{0.17} \mathrm{Se}$ shell. It is present in some NWs.

The higher band gap of $\mathrm{Zn}_{0.83} \mathrm{Mg}_{0.17}$ Se shell prevents the charge carriers to recombine nonradiatively on the $\mathrm{ZnSe}$ NW sidewall and hence improves the quantum yield of the CdSe emitter. In principle, it could directly be used to grow a photonic wire of diameter $\sim \lambda / n_{\mathrm{ZnSe}}$ around the NWQD. However, during the epitaxial shell growth two phenomena are competing: the radial growth of the shell around the wurtzite NWs, and the vertical growth of a 2D $\mathrm{Zn}_{0.83} \mathrm{Mg}_{0.17}$ Se layer on the sample surface. The radial shell growth rate is very low because the growth of $\mathrm{ZnSe}$ on $\mathrm{WZ}$ surfaces is not favorable. Because of this low shell growth rate, a tradeoff has to be found to avoid burying the NWs in a $\mathrm{Zn}_{0.83} \mathrm{Mg}_{0.17} \mathrm{Se}$ matrix. As a result, only thin epitaxial shells can be fabricated.

The complexity of creating a thick epitaxial shell is one of the reasons why we fabricate the photonic structure by 

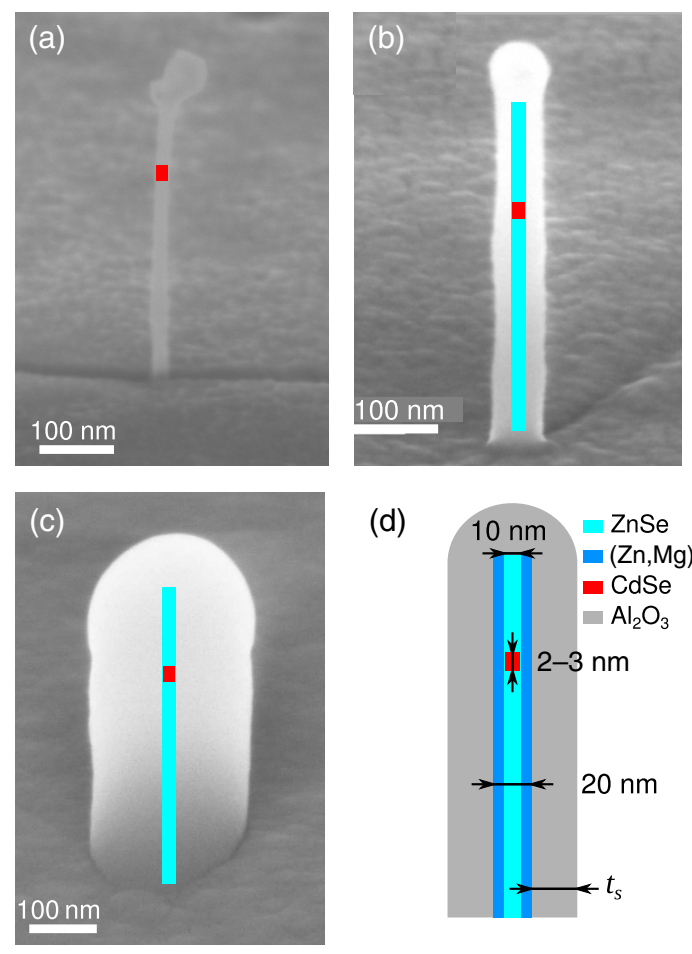

(d)

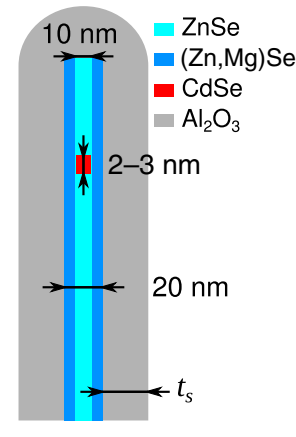

FIG. 2. (a) SEM image of a standing $\mathrm{ZnSe} / \mathrm{Zn}_{0.83} \mathrm{Mg}_{0.17} \mathrm{Se} \mathrm{NW}$ embedding a CdSe QD. The QD position is marked by the red square. (b),(c) Tilted SEM image of a ZnSe NW after a 20-nmthick and 110-nm-thick $\mathrm{Al}_{2} \mathrm{O}_{3}$ shell deposition, respectively. The NW is sketched on the SEM image. Note the circular shape of the shell as well as its hemispherical termination above the NW apex. (d) Sketch of the NWQD geometry, indicating the QD height $(2-3 \mathrm{~nm})$, the NW diameter $(\simeq 10 \mathrm{~nm})$, the epitaxial-shell thickness $(\simeq 5 \mathrm{~nm})$, and the ALD shell thickness $t_{s}$.

depositing an oxide shell around the NW using ALD. Another reason is that, since this process step can be done separately from the NW growth process, it allows one to tune ex situ the shell parameters after a first optical characterization of the QD. Indeed, due to its slow deposition rate, the ALD process allows precise control of the deposited thickness, which can also be finally verified using scanning electron microscopy. We test several oxide materials, and select $\mathrm{Al}_{2} \mathrm{O}_{3}$ because it produces very smooth and conformal, amorphous shells. Figures 2(b) and 2(c) show two SEM images of the resulting oxide-shell deposition (20 and $110 \mathrm{~nm}$ ), and the complete structure is sketched in Fig. 2(d). We note that the conformal deposition allows ending the NW + shell structure by an almost perfect half sphere, as can be seen in Figs. 2(b) and 2(c). ALD also buries the Au droplet under the shell. The latter might interact with the field emitted by the QD through its localized plasmon resonance. Considering its small diameter, it will essentially absorb the incoming field. Moreover, the guided HE11 mode profile presents a minimum on the NW axis. This is why we neglect the droplet influence in the following.

\section{EXPERIMENTAL RESULTS}

A sample from a single epitaxial growth process is cut in pieces, and photonic structures with different oxide-shell thicknesses are fabricated. Taking advantage of the low NW density, individual structures are optically characterized directly on the growth substrate. The samples are mounted on the cold finger of a He-flux cryostat and cooled down to $4 \mathrm{~K}$. Individual photonic structures are probed using confocal microphotoluminescence ( $\mu \mathrm{PL})$. They are excitated by a supercontinuum pulsed laser (Fianium WhiteLase, 10-ps pulse duration, repetition rate $76 \mathrm{MHz}$ ) and a spectrometer selecting a 10-nm bandwidth centered around $485 \mathrm{~nm}$. This excitation energy, below the $\mathrm{ZnSe}$ gap, allows us to induce crossed transitions between delocalized states in the NW 1D continuum and a discrete confined OD state in the NWQD band structure [25]. In this configuration, the NW axis is aligned with the optical axis and emission from the QD is collected by a NA $=0.6$ objective. A typical NWQD spectrum is presented in Fig. 3(a) as a function of the pump laser power. Three lines can be identified and are attributed to the exciton (X), the charged exciton (CX), and the biexciton $(\mathrm{XX})$, respectively. The total emission intensity of the $\mathrm{X}$ line as a function of the pump power is reported in Fig. 3(b). It shows a linear increase at low pumping power, and a constant plateau at high pumping powers corresponding to the saturation of the exciton level [26]. Under pulsed excitation, we note that changing the shell thickness might modify the laser power in the NW and the excitation probability of the QD. Hence, it affects the slope at low power in Fig. 3(b). It has, however, no effect on the saturation plateau which only depends on the QD emission rate and light collection efficiency. This allows us to compare statistical sets of nanostructures with different oxide-shell thicknesses. The total integrated emission at saturation as a function of the oxide-shell thickness is reported in blue markers for each NWQD in Fig. 3(c). The values have been normalized to the average intensity at $t_{s}=110 \mathrm{~nm}$. For NWs without an oxide shell, the luminescence intensity is very low and we were never able to reach the saturation regime, which is why we do not report the corresponding points in Fig. 3(c). For each shell thickness, we observe a large spread in exciton saturation intensity. However, we note a general trend of increasing saturation intensity with increasing shell thickness, as demonstrated by the red markers that show the position of the average intensity of our measurements for each shell thickness. On average, the deposition of a 110-nmthick shell results in the experiments in an almost fourfold enhancement of the collected intensity with respect to the 20-nm-thick shell case. The semianalytical calculations show that this enhancement is tenfold when we compare to a NW without an oxide shell.

The observed increase in intensity at saturation corresponds to the combination of improved collection efficiency through light redirection from the structure and enhancement of the spontaneous emission rate. In the latter 
(a)

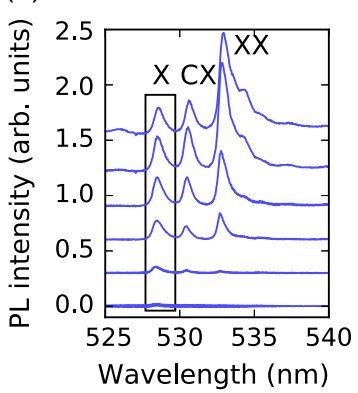

(c)

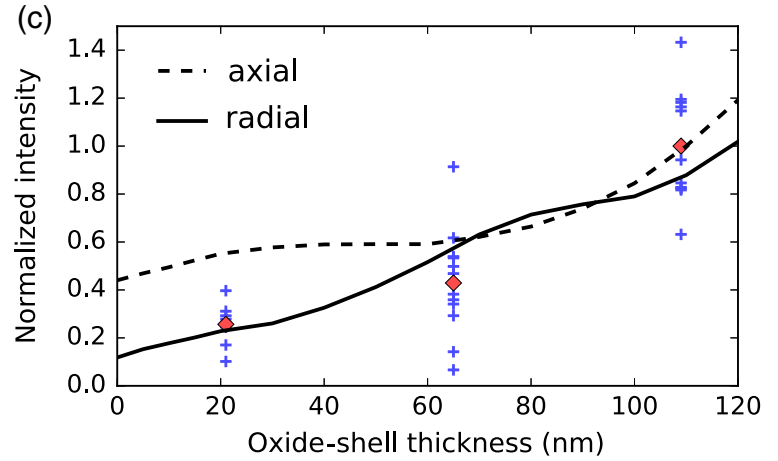

FIG. 3. (a) $\mu \mathrm{PL}$ spectrum of a NWQD with 120-nm-thick photonic shell for different pumping powers. It shows exciton $(\mathrm{X})$, charged exciton (CX), and biexciton (XX) lines. The corresponding pumping powers are reported in panel (b). The black rectangle indicates the integration bandwidth used to extract the total exciton emission intensity (X line). (b) Integrated exciton emission intensity as a function of pumping power, in a $\log -\log$ scale. (c) Blue crosses: Total exciton emission intensity for different NWQDs as a function of the oxide shell radius. Red diamonds: average of the experimental data points. Data are normalized to the average intensity at $t_{s}=110 \mathrm{~nm}$. Black lines: results of the numerical simulations for a radial (solid line) and an axial (dashed line) dipole. They are normalized to the axial intensity at $t_{s}=110 \mathrm{~nm}$.

case, a modification of the QD dynamics is expected to be detected by measuring the exciton decay rate. Timeresolved measurements are carried out using a low pump power as compared to the exciton saturation power to avoid any repopulation of the $\mathrm{X}$ level. The measured decay transients are thus monoexponential. The fitted decay constant is the total exciton decay time $\tau$. The experiment is carried out in another setup on a different set of photonic structures compared to the one of Fig. 3(c). The same excitation laser is used, the QD fluorescence is spectrally filtered in a spectrometer (500 gr/ $\mathrm{mm}$ grating) and integrated on an avalanche photodiode in a photon correlation setup, using the exit slit of the spectrometer as a spectral bandpass filter. The results of these measurements, presented in Fig. 4, show also a great dispersion in decay time. One observes, however, that longer lifetimes are observed for smaller shell thickness (up to $5.9 \mathrm{~ns}$ ). Increasing the shell thickness leads to an overall decrease in the measured exciton lifetime, hence an enhancement of the exciton
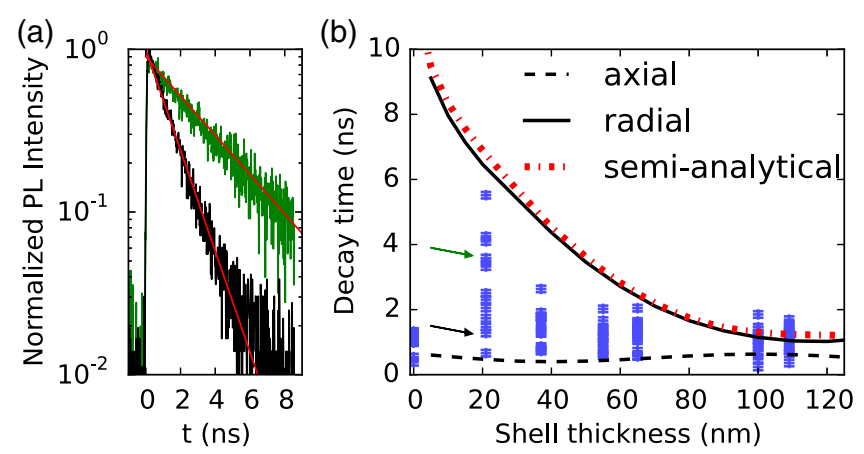

FIG. 4. (a) Example of time-resolved photoluminescence signal versus time for $2 \mathrm{NWs}$ with a $t_{s}=20 \mathrm{~nm}$ shell. Background counts are measured for $t<0$ and substracted. The amplitude of counts are normalized to one to compare the two data sets. Red lines are monoexponential fits, whose corresponding points in (b) are shown by arrows. (b) Blue crosses are experimental exciton decay times for several QDs as a function of the oxide-shell radius. The vertical error bars represent the fit error. Black lines are numerical simulation results for a radial dipole (solid line), or an axial dipole (dashed line). The red dash-dotted line shows semianalytical calculations for the infinite NW.

decay rate in agreement with the results of the numerical simulations. For systems without an oxide shell, only a few NWQDs give a large enough signal to be properly measured. They yield a much smaller dispersion of short decay times.

\section{DISCUSSION AND COMPARISON TO NUMERICAL SIMULATIONS}

\section{A. Dispersion of the results}

For each oxide-shell thickness, the large variations of the experimental results in both Figs. 3(c) and 4 have several possible origins. First, the presence of nonradiative recombination channels can reduce the intensity at saturation and change the decay time. The nonradiative recombination rate can vary from QD to QD because of fabrication inhomogeneities, leading to a spread in the measured values [28]. Second, variations in the QD aspect ratios, and piezoelectric field originating from the strain applied by both the $\mathrm{ZnSe}$ core and the $\mathrm{Zn}_{0.83} \mathrm{Mg}_{0.17}$ Se shell lead to a different overlap of electron and hole wave functions, and hence different exciton oscillator strengths. Finally, considering the QD aspect ratio and internal strain, we expect a heavyhole exciton type for our QDs [29-32]. Heavy-hole exciton recombination results in a mixture of circularly polarized emission, composed of two degenerate out-of-phase radial dipoles. However, strain and confinement effects might lead to valence band mixing between light-hole and heavyhole levels [33-35], resulting in an emission composed of a mixture between axial and radial dipoles and hence to a spread in total emitted intensity, as we discuss later. Additional measurements on NWQDs grown in similar conditions and mechanically dispersed on a substrate 
(i.e., lying horizontally on it) reveal that one NWQD out of six emit light polarized along the NW axis, while others emit light polarized perpendicularly to the $\mathrm{NW}$ axis, showing evidence of the presence of both kinds of dipoles. Because of the $\mathrm{Zn}_{0.83} \mathrm{Mg}_{0.17}$ Se shell and low temperature of observation, we expect that nonradiative effects play a minor role. The epitaxial shell prevents nonradiative decay channels owing to surface traps. Additional measurements as a function of temperature show that both the emission intensity and the decay time do not change significantly up to $150-200 \mathrm{~K}$ (not presented here). This indicates that the nonradiative effects are not dominating at low temperature, as in the present experiment. While we cannot yet completely rule out the contribution of nonradiative effects, we think that the major effect to explain the dispersion of the results comes from variations in valence band mixing and oscillator strength due to the local environment of the QD. Finally, let us stress that the shortest decay times (1-2 ns) we measure remain longer than the decay time of $\mathrm{CdSe}$ self-assembled QD embedded in bulk ZnSe ( $<1 \mathrm{~ns})$ [36]. The reduction of the dielectric screening effect is a main effect we evidence.

\section{B. Collected intensity and radiative lifetime}

To better understand the effect of the shell deposition on the NWQD emission, we perform numerical simulations of the photonic structure formed by the full NW + oxide-shell geometry [see Fig. 2(d)]. It takes into account the presence of the $\mathrm{ZnSe}$ substrate, and the $\mathrm{Al}_{2} \mathrm{O}_{3}$ shell and layer deposited on the NWs and substrate. The QD is modeled as an oscillating electric dipole, either in the axial direction (along the NW axis) or in the radial direction (orthogonal to the NW axis). We perform finite-element method simulations (COMSOL v4.1) to compute the total field radiated by the dipole [35]. For each shell thickness and dipole orientation, we evaluate the power radiated towards the objective by computing the flux of the Poynting vector over a surface limited by its numerical aperture $(\mathrm{NA}=0.6)$ in a region far from the NW where near field can be neglected. The results of these simulations are reported in Fig. 3(c) in black lines for an axial (dashed line) or radial (solid line) dipole. The results are normalized to the axial intensity at $t_{s}=110 \mathrm{~nm}$. Comparing the simulated integrated intensity in the case of $20 \mathrm{~nm}$ and $110 \mathrm{~nm}$ reveals an enhancement factor less than twofold for an axial dipole and almost fourfold for a radial dipole. The fourfold enhancement observed in our measurements suggests that, on average, the dominant emitting dipole in our structure is radial, in good agreement with the recombination of a heavy hole exciton.

The theoretical limits for the radiative lifetimes are extracted from the numerical simulations by integrating the total power radiated over every direction for the two dipole orientations (radial and axial) $P$. We normalize this value by the same quantity computed for a dipole in bulk
ZnSe $P_{0}$. For a purely radiative system we have $P / P_{0}=$ $\gamma / \gamma_{0}=\tau_{0} / \tau$ [37], where $\tau$ and $\tau_{0}$ are the radiative lifetime for the nanostructure and for bulk $\mathrm{ZnSe}$, respectively. Radiative times are presented in black lines in Fig. 4, where we have chosen $\tau_{0}=300 \mathrm{ps}$ in good agreement with the previously reported radiative lifetime of CdSe QD in bulk $\mathrm{ZnSe}$ [38]. The axial dipole radiates with an almost constant decay time as a function of the oxide-shell thickness, while the radial dipole decay time strongly decreases with an increasing oxide-shell thickness $t_{s}$. Additionally, we compare the decay time for the radial dipole computed for the full geometry to the semianalytical calculations for the infinite NW presented in Fig. 1(b) with the same $\tau_{0}$ value. The agreement is excellent indicating that interference effects due to reflections from the substrate and from the top hemispherical termination are negligible.

Comparing the trends of the simulations, we can confirm that our emitters bear a strong radial dipole character. The measurement dispersion can be well understood by considering that the real emitters are a mixture of radial and axial dipoles radiating with a characteristic decay time comprised between the simulated lifetimes of the pure radial and axial dipole. We do not observe a long decay time for NWQDs without an oxide shell in Fig. 4. For these systems, it is very difficult to find emitters which are bright enough to be detected is because both the laser absorption and the emission rate of a radial dipole are very weak for such small NW diameters. We think that the emitters which have been selected correspond to NWQDs having a large fraction of axial dipole character as they are the brightest ones when no oxide shell is present.

\section{Radiation pattern}

To analyze the mechanisms leading to the increase in collected intensity with increasing shell thickness, we present in Fig. 5 several simulated radiation patterns. They are represented as polar plots of the far-field intensity $I(\theta)$ in the top $(x, z)$ plane, $\theta$ is the angle between the direction of observation and the vertical $z$ axis. Simulations are made using, respectively, a radial dipole [along $x$, Figs. 5(a)-5(c)] or an axial dipole [along $z$, Figs. 5(d)-5(f)].

Figures 5(a) and 5(d) show the effect of the NW structure alone (no oxide shell being present) on such dipoles by comparing it to the case of a freestanding dipole in vacuum above the same $\mathrm{ZnSe}$ substrate. One can see that the presence of the NW does not affect the shape of the radiation diagram, which is essentially determined by the interferences between the directly radiated field and its reflection on the substrate. Most remarkably, in the case of the radial dipole the presence of the NW dramatically reduces the emission intensity through the dielectric screening effect discussed earlier. Simulations show a radiative rate reduction by a factor $\sim 1 / 16 \simeq n_{\mathrm{ZnSe}} / 45$ in agreement with the dielectric screening value predicted by Eq. (1). In contrast, in the case of the 


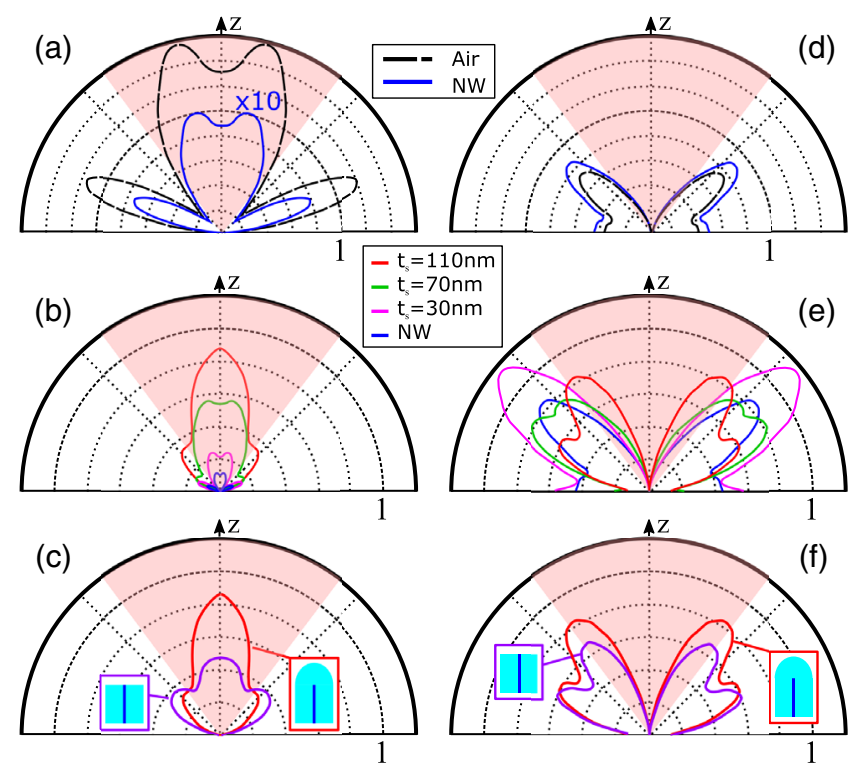

FIG. 5. Radiation patterns from numerical simulations for a radial dipole placed at $470 \mathrm{~nm}$ above the substrate. The experimental NA region is shaded and indicated in red. (a),(d) Comparison between the case of a freestanding emitter in air (black dashes), and embedded inside the NW (blue solid line) for a radial (a) or axial (d) dipole. They evidence the effect of the dielectric screening from the $\mathrm{NW}$ on the radial dipole and the absence of screening for the axial dipole. (b),(e) Comparison of the total emitted intensity versus $t_{s}$ for a radial (b) or axial (e) dipole. A combined effect of reduced dielectric screening and light guiding and redirection towards small angles is observed. (c),(f) Effect of the shell layer termination shape for a radial (c) or axial (f) dipole. The hemispherical shape increases the fraction of light that is redirected towards the $z$ direction.

axial dipole it can be seen that the presence of the NW only slightly increases the emitted intensity.

Figures 5(b) and 5(e) show the computed radiation patterns of the NWQD for increasing oxide-shell thickness $t_{s}$. In the case of the radial dipole, the shell first reduces the index contrast between the NW and the surrounding medium [cf. Eq. (1)], resulting in a strong reduction of the emitter lifetime and thus in an increased total emitted intensity as seen in Figs. 3(c) and 4. Note that the intensity pattern shown in the polar plot must be multiplied by the solid angle $\sin \theta d \theta$ if one wants to evaluate the power radiated in the numerical aperture. This is why the intensity for an axial dipole can be larger than for a radial one, as seen in Fig. 3(c). Second, as shown in Fig. 1(c), the shell presence ensures preferential emission into the guided $\mathrm{HE}_{11}$ mode for increasing shell thickness. As a consequence, a near-Gaussian far-field emission pattern corresponding to the far-field emission profile of the $\mathrm{HE}_{11}$ mode [39] is observed for $t_{s}=110 \mathrm{~nm}$, contrary to the structures with a smaller oxide shell thickness where one observes the presence of two closely spaced lobes at small emission angles $\left( \pm 10^{\circ}\right.$ with respect to the $z$ axis). The resulting emission into the 0.6 NA cone is maximum for $t_{s}=110 \mathrm{~nm}$, where the emission into the $\mathrm{HE}_{11}$ mode is nearly maximum [cf. Fig. 1(b)]. The effect of the oxideshell thickness on the axial dipole is completely different. While the total emitted intensity does not vary much, and hence the emitter lifetime stays constant (as noted in Fig. 4), the light emitted by the axial dipole does not couple to the $\mathrm{HE}_{11}$ mode but is emitted exclusively into radiation modes. Thus, the fraction of intensity emitted towards the collection lens increases only slightly as the oxide-shell thickness increases [cf. Fig. 5(e)]. This intensity increase for the axial dipole also presented in Fig. 3(c) is not due to a change in the spontaneous emission rate of the emitter, but rather to a slight redirection of the emitted light.

Finally, Figs. 5(c) and 5(f) compare the actual hemispherical geometry of the oxide-shell termination to the flat end of a simple lateral shell. They show that the presence of the hemisphere is beneficial to the radiation pattern for both kinds of dipole. For the radial dipole, the hemisphere enables a near-adiabatic expansion of the $\mathrm{HE}_{11}$ mode [39] leading to a narrowing of the far-field emission pattern and an increased collection by the numerical aperture. The axial dipole benefits less from the hemispherical termination of the photonic structure since no light from this dipole is coupled to the HE11 mode. We also note that half of the emitted light propagates towards the growth substrate and due to the index-matching condition between the NW and the substrate, this light is predominantly lost.

In order to assess the performances of our device we compute the ratio $\eta$ between the power radiated into a 0.6 NA to the one radiated into the top air side hemisphere. This parameter is a good figure of merit for the antenna redirection effect although it cannot be directly related to the overall collection efficiency because of the power lost in the substrate. For our full photonic structure and a radial dipole one has $\eta \simeq 80 \%$ for $t_{s}=110 \mathrm{~nm}$. This value reduces to $\simeq 66 \%$ for a flat terminated core-shell photonic wire illustrating the importance of the adiabatic expansion of the $\mathrm{HE}_{11}$ guided mode at the end of the wire. For the dipole in the NW without shell $\eta \simeq 55 \%$. We have also simulated a structure inspired by state-of-the-art devices fabricated by top-down methods in Ref. [9]. In this case we simulate a 110 -nm oxide-shell photonic wire where the hemispherical termination is replaced by a conical tapper of $\mathrm{Al}_{2} \mathrm{O}_{3}$ whose radius progressively decreases from 120 to $10 \mathrm{~nm}$ in $1.5 \mu \mathrm{m}$. In this case one has $\eta \simeq 94 \%$, showing that although beneficial our hemispherical termination is not optimal.

\section{CONCLUSION}

In summary, we present a bottom-up approach to fabricating a dielectric antenna around a QD inserted inside a NW. This method allows for both reproducible and very precise fabrication of the structure on a large ensemble of emitters at once. It is based on the deposition of a thick 
oxide shell around the NW using atomic-layer deposition. Experiments show a fourfold enhancement of the QD photoluminescence shown in Fig. 3(c) between a 20-nmthick shell and a 110-nm-thick shell. Semianalytical calculations and numerical simulations of the structure reveal that the oxide-shell thickness strongly acts on the radial dipole emission through two main phenomena: the reduction of the dielectric screening, which increases the spontaneous emission rate from the QD, and the redirection of light through a waveguiding effect. Simulations suggest that the collected intensity is multiplied by a factor 7 with respect to the bare NW case. The fabrication process of the photonic shell is very simple and can be applied to QDs emitting single photons up to room temperature. Although not optimal, the resulting structure is a step towards the best nanowire single-photon sources operating at low temperature [9]. Dielectric screening could be further reduced by growing an oxide shell of higher index matching $n_{\mathrm{ZnSe}}$ like $\mathrm{TiO}_{2}$. We note also that in our system a large fraction of the emitted power is radiated in the substrate. This loss channel could be reduced by having a mirror at the bottom of the structure $[15,40]$. Moreover, to fully benefit from the waveguiding approach, a better control on the intrinsic QD properties has to be reached to ensure the presence of radial dipoles, which radiate more efficiently in the experimental collection aperture.

\section{ACKNOWLEDGMENTS}

This work was supported by the French National Research Agency under Contract No. ANR-10-LABX51-01 and the Danish Research Council for Technology and Production (LOQIT Sapere Aude Grant No. DFF 4005-00370).

M. J. and T. C. contributed equally to this work.

[1] P. Michler, A. Kiraz, C. Becher, W. V. Schoenfeld, P. M. Petroff, L. Zhang, E. Hu, and A. Imamoglu, A quantum dot single-photon turnstile device, Science 290, 2282 (2000).

[2] Charles Santori, Matthew Pelton, Glenn Solomon, Yseulte Dale, and Yoshihisa Yamamoto, Triggered Single Photons from a Quantum Dot, Phys. Rev. Lett. 86, 1502 (2001).

[3] Charles Santori, David Fattal, Jelena Vučković, Glenn S. Solomon, and Yoshihisa Yamamoto, Indistinguishable photons from a single-photon device, Nature (London) 419, 594 (2002).

[4] A. Zrenner, E. Beham, S. Stufler, F. Findeis, M. Bichler, and G. Abstreiter, Coherent properties of a two-level system based on a quantum-dot photodiode, Nature (London) 418, 612 (2002).

[5] N. Akopian, N. H. Lindner, E. Poem, Y. Berlatzky, J. Avron, D. Gershoni, B. D. Gerardot, and P. M. Petroff, Entangled Photon Pairs from Semiconductor Quantum Dots, Phys. Rev. Lett. 96, 130501 (2006).
[6] Andrew J. Shields, Semiconductor quantum light sources, Nat. Photonics 1, 215 (2007).

[7] E. Viasnoff-Schwoob, C. Weisbuch, H. Benisty, S. Olivier, S. Varoutsis, I. Robert-Philip, R. Houdré, and C. J. M. Smith, Spontaneous Emission Enhancement of Quantum Dots in a Photonic Crystal Wire, Phys. Rev. Lett. 95, 183901 (2005).

[8] T. Lund-Hansen, S. Stobbe, B. Julsgaard, H. Thyrrestrup, T. Sünner, M. Kamp, A. Forchel, and P. Lodahl, Experimental Realization of Highly Efficient Broadband Coupling of Single Quantum Dots to a Photonic Crystal Waveguide, Phys. Rev. Lett. 101, 113903 (2008).

[9] Julien Claudon, Joël Bleuse, Nitin Singh Malik, Maela Bazin, Périne Jaffrennou, Niels Gregersen, Christophe Sauvan, Philippe Lalanne, and Jean-Michel Gérard, A highly efficient single-photon source based on a quantum dot in a photonic nanowire, Nat. Photonics 4, 174 (2010).

[10] J. Heinrich, A. Huggenberger, T. Heindel, S. Reitzenstein, S. Höfling, L. Worschech, and A. Forchel, Single photon emission from positioned GaAs/AlGaAs photonic nanowires, Appl. Phys. Lett. 96, 211117 (2010).

[11] Joël Bleuse, Julien Claudon, Megan Creasey, Nitin. S. Malik, Jean-Michel Gérard, Ivan Maksymov, Jean-Paul Hugonin, and Philippe Lalanne, Inhibition, Enhancement, and Control of Spontaneous Emission in Photonic Nanowires, Phys. Rev. Lett. 106, 103601 (2011).

[12] Mathieu Munsch, Julien Claudon, Joël Bleuse, Nitin S. Malik, Emmanuel Dupuy, Jean-Michel Gérard, Yuntian Chen, Niels Gregersen, and Jesper Mørk, Linearly Polarized, Single-Mode Spontaneous Emission in a Photonic Nanowire, Phys. Rev. Lett. 108, 077405 (2012).

[13] Mathieu Munsch, Nitin S. Malik, Emmanuel Dupuy, Adrien Delga, Joël Bleuse, Jean-Michel Gérard, Julien Claudon, Niels Gregersen, and Jesper Mørk, Dielectric GaAs Antenna Ensuring an Efficient Broadband Coupling between an InAs Quantum Dot and a Gaussian Optical Beam, Phys. Rev. Lett. 110, 177402 (2013).

[14] E. M. Purcell, in Proceedings of the American Physical Society (American Physical Society (APS), College Park, MD, 1946), Vol. 69, p. 674.

[15] Michael E. Reimer, Gabriele Bulgarini, Nika Akopian, Moïra Hocevar, Maaike Bouwes Bavinck, Marcel A. Verheijen, Erik P. A. M. Bakkers, Leo P. Kouwenhoven, and Val Zwiller, Bright single-photon sources in bottom-up tailored nanowires, Nat. Commun. 3, 737 (2012).

[16] Gabriele Bulgarini, Michael E. Reimer, Tilman Zehender, Moïra Hocevar, Erik P. A. M. Bakkers, Leo P. Kouwenhoven, and Valery Zwiller, Spontaneous emission control of single quantum dots in bottom-up nanowire waveguides, Appl. Phys. Lett. 100, 121106 (2012).

[17] G. Bulgarini, M. E. Reimer, M. B. Bavinck, K. D. Jöns, D. Dalacu, P. J. Pool, E. P. Bakkers, and V. Zwiller, Nanowire waveguide launching single photons in a Gaussian mode for ideal fiber coupling, Nano Lett. 14, 4102 (2014).

[18] Adrien Tribu, Gregory Sallen, Thomas Aichele, Régis André, Jean-Philippe Poizat, Catherine Bougerol, Serge Tatarenko, and Kuntheak Kheng, A high-temperature single-photon source from nanowire quantum dots, Nano Lett. 8, 4326 (2008). 
[19] S. Bounouar, M. Elouneg-Jamroz, M. den Hertog, C. Morchutt, E. Bellet-Amalric, R. André, C. Bougerol, Y. Genuist, J.-Ph. Poizat, S. Tatarenko, and K. Kheng, Ultrafast room temperature single-photon source from nanowirequantum dots, Nano Lett. 12, 2977 (2012).

[20] Y.-R. Nowicki-Bringuier, R. Hahner, J. Claudon, G. Lecamp, P. Lalanne, and J.-M. Gérard, A novel highefficiency single-mode single photon source, Ann. Phys. (Paris) 32, 151 (2008).

[21] T. Cremel, M. Elouneg-Jamroz, E. Bellet-Amalric, L. Cagnon, S. Tatarenko, and K. Kheng, Bottom-up approach to control the photon outcoupling of a II-VI quantum dot with a photonic wire, Phys. Status Solidi C 11, 1263 (2014).

[22] Julien Claudon, Niels Gregersen, Philippe Lalanne, and Jean-Michel Gérard, Harnessing light with photonic nanowires: Fundamentals and applications to quantum optics, ChemPhysChem 14, 2393 (2013).

[23] A. Yariv, Optical Electronics in Modern Communications (Oxford University Press, Oxford, 1997).

[24] Teppo Häyrynen, Jakob Rosenkrantz de Lasson, and Niels Gregersen, Open-geometry Fourier modal method: Modeling nanophotonic structures in infinite domains, J. Opt. Soc. Am. A 33, 1298 (2016).

[25] A. Vasanelli, R. Ferreira, and G. Bastard, Continuous Absorption Background and Decoherence in Quantum Dots, Phys. Rev. Lett. 89, 216804 (2002).

[26] We note that single-photon emission is preserved if one integrates both of the signals from the $\mathrm{X}$ and CX lines [27].

[27] G. Sallen, A. Tribu, T. Aichele, R. André, L. Besombes, C. Bougerol, S. Tatarenko, K. Kheng, and J. Ph. Poizat, Exciton dynamics of a single quantum dot embedded in a nanowire, Phys. Rev. B 80, 085310 (2009).

[28] Petr Stepanov, Adrien Delga, Xiaorun Zang, Joël Bleuse, Emmanuel Dupuy, Emanuel Peinke, Philippe Lalanne, Jean-Michel Gérard, and Julien Claudon, Quantum dot spontaneous emission control in a ridge waveguide, Appl. Phys. Lett. 106, 041112 (2015).

[29] J. D. Eshelby, The determination of the elastic field of an ellipsoidal inclusion, and related problems, Proc. R. Soc. A 241, 376 (1957).
[30] J. D. Eshelby, The elastic field outside an ellipsoidal inclusion, Proc. R. Soc. A 252, 561 (1959).

[31] M. Zieliński, Fine structure of light-hole excitons in nanowire quantum dots, Phys. Rev. B 88, 115424 (2013).

[32] David Ferrand and David Fattal Joël Cibert, Strain in crystalline core-shell nanowires, Eur. Phys. J. Appl. Phys. 67, 30403 (2014).

[33] K. F. Karlsson, V. Troncale, D. Y. Oberli, A. Malko, E. Pelucchi, A. Rudra, and E. Kapon, Optical polarization anisotropy and hole states in pyramidal quantum dots, Appl. Phys. Lett. 89, 251113 (2006).

[34] Catherine Tonin, Richard Hostein, Valia Voliotis, Roger Grousson, Aristide Lemaitre, and Anthony Martinez, Polarization properties of excitonic qubits in single self-assembled quantum dots, Phys. Rev. B 85, 155303 (2012).

[35] Mathieu Jeannin, Alberto Artioli, Pamela Rueda-Fonseca, Edith Bellet-Amalric, Kuntheak Kheng, Régis André, Serge Tatarenko, Joël Cibert, David Ferrand, and Gilles Nogues, Light-hole exciton in a nanowire quantum dot, Phys. Rev. B 95, 035305 (2017).

[36] G. Bacher, R. Weigand, J. Seufert, V. D. Kulakovskii, N. A. Gippius, A. Forchel, K. Leonardi, and D. Hommel, Biexciton versus Exciton Lifetime in a Single Semiconductor Quantum Dot, Phys. Rev. Lett. 83, 4417 (1999).

[37] L. Novotny and B. Hecht, Principles of Nano-Optics, 2nd ed. (Cambridge University Press, Cambridge, 2012).

[38] T. Flissikowski, A. Hundt, M. Lowisch, M. Rabe, and F. Henneberger, Photon Beats from a Single Semiconductor Quantum Dot, Phys. Rev. Lett. 86, 3172 (2001).

[39] Niels Gregersen, Torben R. Nielsen, Julien Claudon, JeanMichel Gérard, and Jesper Mørk, Controlling the emission profile of a nanowire with a conical taper, Opt. Lett. 33, 1693 (2008).

[40] I. Friedler, P. Lalanne, J. P. Hugonin, J. Claudon, J. M. Gérard, A. Beveratos, and I. Robert-Philip, Efficient photonic mirrors for semiconductor nanowires, Opt. Lett. 33, 2635 (2008). 